\title{
Respiration apparatus comprising fixing string: a new design of double (head and neck) straps for patient face mask
}

\author{
Sang-Kyi Lee and A Ram Doo \\ Department of Anesthesiology and Pain Medicine, Chonbuk National University Medical School, Jeonju, Korea
}

The oxygen delivery to hypoxemic patients is mandatory via a number of different types of oxygen masks such as a simple face mask, a partial rebreathing mask, a non-rebreathing mask and a Venturi mask. The conventional masks, which cover the nose and the mouth, are usually made of plastic and fixed on the face by a single elastic head strap. If the oxygen mask fits loosely on the face, much inhaled room air mixed with the supplied oxygen may enter the partial rebreathing mask and the nonrebreathing mask. Consequently, the inspired oxygen concentration of inhaled gas may decrease. Therefore, tight mask-fitting and good mask-sealing are critical to receive adequate amount of oxygen undiluted by room air in severely hypoxemic patients. There is only one single head strap for conventional masks that passes by or is on the external ear and fixed on the occipital area of the patients. The head strap may damage the skin near the external ear for through prolonged period of use and cause the patient's discomfort. In particular, the skin near the external ear can be easily injured by slipping and moving straps when the patients are very irritable.

We have devised two elastic straps to secure the fitting and the sealing of the mask and prevent the skin on the external ear from irritation (Fig. 1). The first strap is one for the head. The newly revised head strap for an oxygen mask is composed of two elastic strands. We have split one-third mid-portion of a conventional single head strap longitudinally to double strands to fix tightly between the anterior/posterior portion of the top of the patient's head and the upper part of the face mask. The rest two-thirds of the new head strap are one strand. The second strap is one for the neck. We have added the neck strap to fix tightly between the neck and the lower part of the oxygen mask.

Face masks can be placed tightly and securely without any slip or displacement of the head strap towards the external ear with the newly revised two separated straps. And the complication from the conventional mask such as skin abrasion and irritation near the external ear can be reduced, especially for prolonged use. In addition, the inspired oxygen concentration

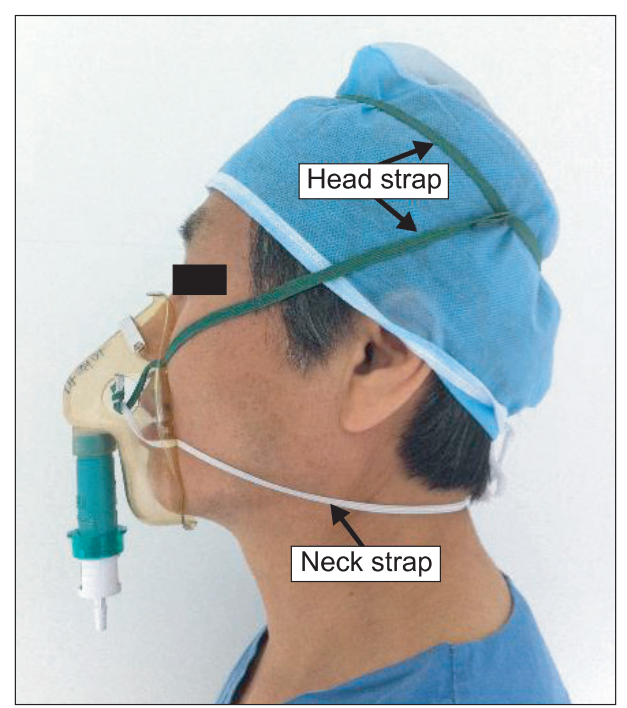

Fig. 1. A picture of the patient wearing the oxygen mask with newly designed double straps for face masks, which are consisted of a dually split head strap and a neck strap.

Corresponding author: Sang-Kyi Lee, M.D., Ph.D., Department of Anesthesiology and Pain Medicine, Chonbuk National University Medical School \& Chonbuk National University Hospital, 20, Gunji-ro, Deokjin-gu, Jeonju 561-712, Korea. Tel: 82-63-250-1250, Fax: 82-63-250-1240, E-mail: leesk@jbnu.ac.kr

(c) This is an open-access article distributed under the terms of the Creative Commons Attribution Non-Commercial License (http:// creativecommons.org/licenses/by-nc/3.0/), which permits unrestricted non-commercial use, distribution, and reproduction in any medium, provided the original work is properly cited. 
is accurately and precisely maintained without any dilution of the inspired pure oxygen with room air because of the tight face mask-sealing/fitting.

Two elastic straps may be a little bit cumbersome for doctors and nurses since they have to put the each strap on the patient's head and the neck. However, because the color tone of the head strap (dark green) and the neck strap (white) are different, health care providers using the newly designed straps can make an easy distinction between the head strap and the neck strap without any confusion. 